the Earth spins are caused by seasonal changes in the general circulation of the atmosphere, and he argued that meteorological effects could well be responsible for sudden changes in the LOD as well. He cast doubt on the reliability of evidence that earthquakes or sunspots might be causes of changes in the LOD. This is not to say that the overall trend in the acceleration of the Earth could not be influenced by solar activity, but "it is dangerous to argue a causal relationship between specific solar events and changes in the LOD".

Around the time of the recent Earth spin event, there were, it seems, changes in the pattern of westerly winds at the 500 mbar level, but Thomas declined to be drawn onto dangerous speculative ground, closing his talk with an emphatic statement that he would not attempt to say what causes the meteoro. logical variations. As readers of the News and Views section will be aware, however, there is now compelling evidence that solar activity affects the very meteorological processes which seem to affect the LOD (see Nature, 246, 384; 1973).

\section{Earth plates as membranes}

from our Geomagnetism Correspondent IN recent years several workers have sought to explain the origin of major tectonic activity away from plate boundaries in terms of hot spots, with or without associated mantle plumes. The best known example of the use of such an interpretation is probably the Hawaiian-Emperor island chain which is seen as the surface trace resulting from the motion of the Pacific plate over a stationary or near stationary magma source; but continental graben and rift valleys, such as the Rhine graben, have also been associated with hot spots. On the other hand, there are problems with this viewpoint and there are alternative, if no less contentious, explanations for both continental and oceanic mid-plate tectonic structures. The Rhire graben, for example, has an average width of $36 \mathrm{~km}$ and an estimated extension across the valley of $4.8 \mathrm{~km}$; but it is not entirely clear how such features could result from hot spot or plume activity. And as far as the formation of the Hawaiian islands is concerned, the hypothesis that the chain derives from a propagating tensional fracture in the lithosphere which causes volcanic activity as it extends was put forward as early as 1942 and still receives much support today.

The chief difficulty with both the older and newer interpretations of the Hawaiian chain lies in substantiating them, although the former was recently given a significant boost by Turcotte and Oxburgh (Nature, 244, 337; 1973) who offered two possible origins for the required tensional stresses. The first of these involved thermal effects. Lithosphere created at an oceanic ridge by the cooling of mantle material cools further and increases in thickness as it moves away from the ridge. Such an elastic plate cooled non-uniformly will be subject to thermal stresses in the form of tensions which are parallel to the ridge, and thus aligned with the corresponding magnetic anomalies, but normal to fracture zones. Turcotte and Oxburgh were able not only to calculate the thermal stresses and show them to be sufficient to fracture the lithosphere but also to predict the direction of the tensional thermal stresses near Hawaii and show it to be in close agreement with a similar prediction using the theory for the plastic yielding and fracture of elastic solids.

The second type of tensional stress, the theory of which has now been developed at greater length by Turcotte (Geophys. J., 36, 33; 1974), is the membrane stress arising from non-spherical plate tectonics. The basic point here is that if the Earth were a perfect sphere plates would be able to move about without deformation but, because the Earth is in fact an oblate spheroid with an ellipticity of 0.00335 , the lithosphere must deform when its latitude changes. A small plate at the equator, for example, will have two principal radii of curvature $(6,378 \mathrm{~km}$ and $6,335 \mathrm{~km})$, both of which will increase if the plate moves towards higher latitudes and both of which reach $6,400 \mathrm{~km}$ for a plate at either of the poles. Conversely, a small plate moving towards the equator will have its principal radii of curvature decreased. Normally such changes in the radii of curvature would produce bending stresses, but becau'se the surface plates in this case are thin compared with the Earth's radius they will behave as thin shells in which the bending stresses may be neglected in comparison with the membrane stresses arising from stretching the plates to different radii of curvature.

In principle, therefore, it should be possible to determine the membrane stresses associated with the deformation of the Earth's moving plates using the already developed theory of thin shells -and this is what Turcotte has tried to do. For an unstressed thin shell whose radii of curvature are increased, the result will be a tension at the edge and a compression in the interior; for a decrease in the radii of curvature the situation will be reversed. But, first, do such membrane stresses actually exist in real plates? Plates move with velocities in the range $1-10 \mathrm{~cm} \mathrm{yr}^{-1}$, thus requiring about $10^{8} \mathrm{yr}$ to undergo a significant change in latitude. It is usual to regard the lithosphere as an elastic medium on a short time scale; but on the geological time scale will plastic yielding occur, relieving the membrane stresses? As Turcotte points out, at "modest" depths plastic yielding is to be expected because of the higher temperatures involved. But he also notes that the relief of membrane stresses requires plastic flow throughout the who'e thickness of a plate and that there is abundant evidence (for example, from major fault systems) for the absence of long term plastic flow in near surface rocks.

Having disposed of this point (which is not the Aunt Sally it might at first seem), Turcotte goes on to develop the theory of membrane tectonics, generally along the lines mapped out by Novozhilov (The theory of Thin Shells, Noordhoff, 1959). For the real Earth the determination of the membrane stresses is, of course, an insolubly complex problem; surface plates have irregular shapes and each element of each large plate has its own radii of curvature which change in accordance with the particular variation in latitude. Turcotte thus adopts a highly simplified model in which the initially unstressed plate is represented by a circular segment of a spherical shell and in which the deformed plate is a similar spherical dome but with a slightly larger or smaller radius of curvature. In this scheme, deformation is produced by a radial surface force on the shell.

What emerges from this anaiysis is that a change in the latitude of the modelied lithosphere can result in membrane stresses of up to several kilobars. The stress required to produce a fracture in the real lithosphere is not precisely known, although stresses on active fault zones are known to be of the order of $0.1 \mathrm{kbar}$ and laboratory work has shown the strength of mantle rocks to be of the order of $10 \mathrm{kbar}$. It may thus be fairly safely presumed that membrane stresses of several kilobars would indeed be sufficient to fracture the lithosphere, although thermal stresses and the stresses driving the plates may also play a part. Turcotte further shows that membrane stresses of about $2 \mathrm{kbar}$ should lead to gravity anomalies of the order of $10 \mathrm{mgal}$, which is the magnitude of the anomalies actually observed.

Whether or not the importance of membrane stress is substantiated, Turcotte's analysis can only strengthen the hand of workers such as Jackson and Wright (Petrology, 11, 405; 1970) who see the Hawaiian chain in terms of a propagating fracture rather than a hot spot. Moreover, membrane stresses seem to be the more capable of explaining the finite extension of rift valleysas the extension needed to relieve the stresses. 